\title{
Desenvolvimento populacional de duas espécies de cladóceros alimentados com microalgas cultivadas em laboratório
}

\author{
Population development of two cladoceran species fed on laboratory-grown \\ microalgae
}

DOI: $10.46814 /$ lajdv3n3-019

Recebimento dos originais: 01/05/2021

Aceitação para publicação: 30/06/2021

\section{Lucas Henrique Cortat}

Graduado em Aquicultura pelo IFES. Aluno do curso de pós-graduação em ciências veterinárias (PPGCV) na Universidade Federal do Espírito Santo - Campus Alegre, 2021/01.

E-mail: lucascortat@gmail.com

\begin{abstract}
RESUMO
O presente estudo avalia o efeito da dieta sobre a reprodução e o desenvolvimento dos cladóceros Ceriodaphnia dubia e Simocephalus iheringi, cultivados em laboratório. Indivíduos adultos foram distribuídos por seis frascos de 1 L (10 indivíduos por frasco), divididos em dois lotes iguais. Um lote foi tratado com dieta mista de microalgas (Ankistrodesmus sp., Dictyosphaerium sp. e Volvox sp.) cultivadas em laboratório e o outro lote foi tratado com dieta unialgal (Volvox sp.). O período experimental foi de 21 dias, com fotoperíodo de $8 \mathrm{~h}$ e temperatura entre 26 e $28{ }^{\circ} \mathrm{C}$. A quantidade fornecida foi de $20 \mathrm{~mL}$ da cultura algal com a densidade de 1,01 x 106 células mL-1, a cada quatro dias. Foram feitas duas contagens da população (sete e 14 dias), em câmara de Sedgwick-Rafter, com aumento de $40 \mathrm{X}$, utilizando-se subamostras de $1 \mathrm{~mL}$. Para cada tratamento foi verificada a fecundidade média, o tempo de desenvolvimento embrionário, o tempo de desenvolvimento pós-embrionário, a idade da primeira reprodução (IPR), o tempo de vida (TV), a taxa intrínseca de crescimento e o tempo de duplicação da população. Para as duas espécies estudadas, a fecundidade média e o crescimento populacional foram maiores, com a dieta mista. Esta proporcionou maior tempo de vida para Ceridaphnia dubia e menor tempo de vida para Simocephalus iheringi, em relação à dieta unialgal. A fecundidade foi afetada pela dieta, pelo tamanho das fêmeas (Ceridaphnia dubia) e pelo tamanho da população (Simocephalus iheringi). Os melhores resultados, para ambas as espécies, foram obtidos com a dieta mista.
\end{abstract}

Palavras-Chave: alimento vivo, cultivo de cladóceros, dieta.

\begin{abstract}
The present study evaluates the effect of diet on the reproduction and development of the cladocerans Ceriodaphnia dubia and Simocephalus iheringi, grown in the laboratory. Adult individuals were distributed into six $1 \mathrm{~L}$ flasks (10 individuals per flask), divided into two equal lots. One batch was treated with a mixed microalgae diet (Ankistrodesmus sp., Dictyosphaerium sp. And Volvox sp.) Grown in the laboratory and the other batch was treated with a unialgal diet (Volvox sp.). The experimental period was 21 days, with a photoperiod of $8 \mathrm{~h}$ and temperature between 26 and $28^{\circ} \mathrm{C}$. The amount supplied was $20 \mathrm{~mL}$ of the algal culture with a density of 1.01 x 106 cells mL-1, every four days. Two population counts were performed (seven and 14 days), in a Sedgwick-Rafter chamber, with a 40X magnification, using $1 \mathrm{~mL}$ subsamples. For each treatment, the average fertility, the time of embryonic development, the time of post-embryonic development, the age of first reproduction (IPR), the time of life (TV), the intrinsic growth rate and the doubling time were checked. of the population. For the two species studied, the average fertility and population growth were higher, with
\end{abstract}


the mixed diet. This provided a longer life span for Ceridaphnia dubia and a shorter life span for Simocephalus iheringi, in relation to the unialgal diet. Fertility was affected by diet, female size (Ceridaphnia dubia) and population size (Simocephalus iheringi). The best results, for both species, were obtained with the mixed diet.

Keywords: live food, cultivation of cladocerans, diet.

\section{INTRODUÇÃO}

A disponibilidade de alimento com alto valor nutricional é de grande relevância para a sobrevivência e o desenvolvimento inicial de pós-larvas de peixes. O zooplâncton é um recurso alimentar adequado, pois, além do alto valor nutricional, contém enzimas digestivas, que estimulam o desenvolvimento do sistema digestório das pós-larvas de peixes.

No Brasil, a prática mais utilizada na larvicultura é a adubação dos viveiros alguns dias antes do povoamento com as pós-larvas, garantindo a disponibilidade de alimento natural (ZANIBONI FILHO, 2000). Entretanto, o tempo necessário para o aparecimento da comunidade planctônica varia entre os viveiros, conforme a qualidade da água, a qualidade dos nutrientes encontrados nela e a temperatura.

A heterogeneidade no crescimento do plâncton em diferentes locais faz com que as orientações técnicas sejam variadas, sendo necessário, portanto, que cada produtor conheça a capacidade de produção planctônica do seu viveiro, para adequar o manejo às condições de sua propriedade (ZANIBONI FILHO, 2000).

Segundo Santeiro et al. (2006), a fertilização é o principaL fator responsável pelo aparecimento da comunidade planctônica. Esta apresenta maior valor nutricional no terceiro dia a partir da adubação, havendo um decréscimo na composição bioquímica, após esse período.

A adubação dos viveiros é benéfica para muitas espécies de peixes, possibilitando o aumento da produtividade. Entretanto, ela também provoca o aumento do nível de fósforo na água. O problema é agravado quando se utiliza adubo orgânico, pois este tem baixa concentração de nutrientes, sendo necessária uma grande quantidade. $\mathrm{O}$ uso excessivo de adubo orgânico pode causar a redução dos níveis de oxigênio e favorecer a proliferação de fungos, bactérias e protozoários parasitas.

Uma alternativa para a solução desses problemas é o cultivo do alimento natural em laboratório ou em tanques ao ar livre, específicos para tal fim. Várias espécies zooplanctônicas, incluindo rotíferos, cladóceros e copépodos calanoides, são cultivadas como alimento para pós-larvas de peixes e de camarões de água doce.

Muitos fatores interferem no desenvolvimento do plâncton cultivado e muitas são as técnicas de cultivo. Portanto é necessário o conhecimento das necessidades nutricionais e dos fatores físicos e 
químicos que afetam o desenvolvimento desses organismos, para a produção em quantidade suficiente e com qualidade adequada para a alimentação das pós-larvas de peixes e de crustáceos (SIPAÚBATAVARES ET AL, 2002).

\section{OBJETIVO}

O presente estudo avalia o efeito da dieta sobre a reprodução e o ciclo de vida dos cladóceros Ceriodaphnia dubia e Simocephalus iheringi criados em laboratório.

\section{MATERIAL E MÉTODOS}

O presente estudo foi realizado no Laboratório de Ecologia Aquática e Produção de Plâncton (LEAPP) do Instituto Federal do Espírito Santo (Ifes) - Campus de Alegre.

Os espécimes utilizados no experimento foram capturados nos viveiros de piscicultura do Ifes - Campus de Alegre, com um balde de 10 L, filtrando-se a água em rede de zooplâncton com malha 65 $\mu \mathrm{m}$. Alguns espécimes foram fixados em formalina tamponada a 4\%, para a identificação, que foi feita com base em Elmoor-Loureiro (1997). Duas espécies foram utilizadas no experimento: Ceriodaphnia dubia e Simocephalus iheringi.

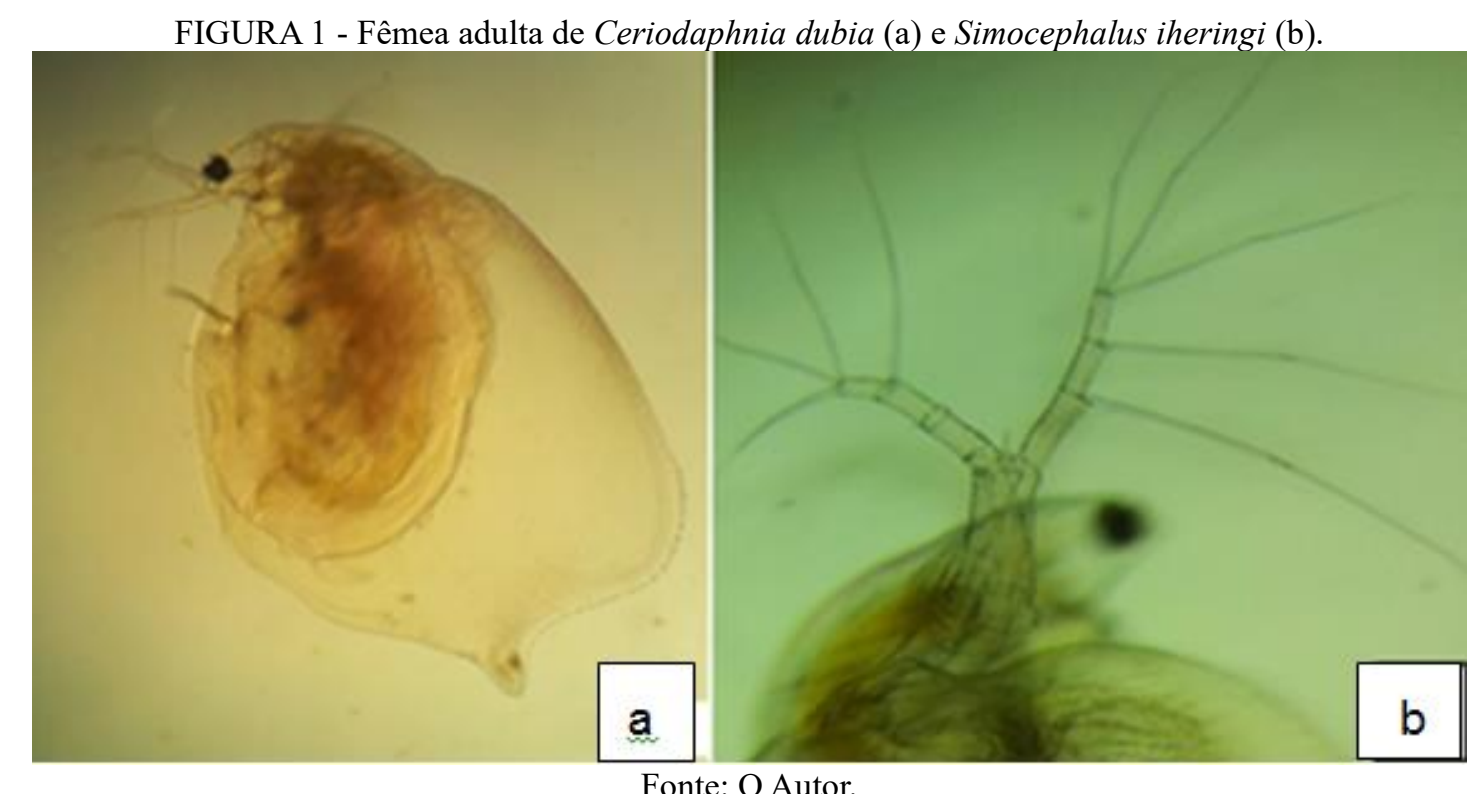

Para a realização do experimento, fêmeas adultas foram distribuídos por 12 frascos de $1 \mathrm{~L}$, divididos em quatro lotes de três. Cada frasco recebeu 10 indivíduos. O período experimental foi de 21 dias, com fotoperíodo de $8 \mathrm{~h}$ diárias. Durante a realização do experimento, a temperatura foi mantida entre 26 e $28^{\circ} \mathrm{C}$ e foi feita a renovação de $20 \%$ da água, a cada 7 dias.

A dieta consistiu em microalgas provenientes de cultura em laboratório, com a densidade de 
$1,01 \times 10^{6}$ células $\mathrm{mL}^{-1}$. Foram utilizadas duas culturas de microalgas: cultura mista de Ankistrodesmus sp., Dictyosphaerium sp. e Volvox sp., e cultura unialgal de Volvox sp. A quantidade fornecida foi de 20 $\mathrm{mL}$ da cultura algal, a cada quatro dias. Foram utilizadas culturas de algas com 15 dias de idade. A contagem do número de células foi feita em câmara de Neubauer. Para evitar a alteração do número de células, após a contagem, as culturas foram guardadas em geladeira, a $4{ }^{\circ} \mathrm{C}$, sendo utilizadas por um período de 14 dias. Após esse período, as culturas foram substituídas, para que não houvesse perda do valor nutricional.

O crescimento populacional dos cladóceros foi acompanhado, fazendo-se a contagem do número de indivíduos, após cada evento reprodutivo. As contagens foram feitas em câmara de Sedgwick-Rafter, sob microscópio estereoscópico, com aumento de 40 X. Para a contagem, amostras de $100 \mathrm{~mL}$ de água eram retiradas dos frascos de cultivo e filtradas em copo para filtração, com fundo de malha $68 \mu \mathrm{m}$.

Para cada tratamento foi verificada a fecundidade média (número de ovos por fêmea), o tempo de desenvolvimento embrionário (TDE), o tempo de desenvolvimento pós-embrionário (TDPE), a idade da primeira reprodução (IPR) e o tempo de vida (TV). O tempo de desenvolvimento embrionário foi determinado pela observação diária de fêmeas adultas, mantidas em placa de Petri com água, sendo uma fêmea em cada placa, com cinco repetições. As fêmeas eram isoladas nas placas de Petri após a liberação de uma ninhada, antes que surgisse a próxima remessa de ovos. A partir da postura dos ovos na câmara incubadora, contavam-se os dias até a liberação dos neonatos.

A taxa intrínseca de crescimento (r) foi determinada pela fórmula $\left(\ln \mathrm{N}_{1}-\ln \mathrm{N}_{0}\right) / t$, onde:

$\mathrm{N}_{1}=$ número de indivíduos após um determinado tempo

$\mathrm{N}_{0}=$ número de indivíduos no início do cultivo

$\mathrm{t}=$ tempo de cultivo

O tempo de duplicação (d) da população foi determinado pela fórmula ln 2/r.

Os dados de crescimento populacional foram submetidos à análise de variância e as médias foram comparadas pelo teste de Tukey, em nível de 5\% de significância.

\section{RESULTADOS E DISCUSSÃO}

Para as duas espécies estudadas, o crescimento populacional foi maior, no tratamento com dieta mista. A diferença no crescimento da população, entre os tratamentos, foi mais acentuada para Ceriodaphnia dubia do que para Simocephalus iheringi, embora a diferença entre os tratamentos tenha sido significativa $(\mathrm{P} \leq 0,05)$ para ambas as espécies (Gráficos 1 e 2). 
Gráfico 1 - Crescimento populacional de Ceriodaphnia dubia alimentada com cultura mista (CM) e com Volvox sp. (V) ( $\mathrm{R} 1$ = primeiro evento reprodutivo; $\mathrm{R} 2$ = segundo evento reprodutivo $)$.

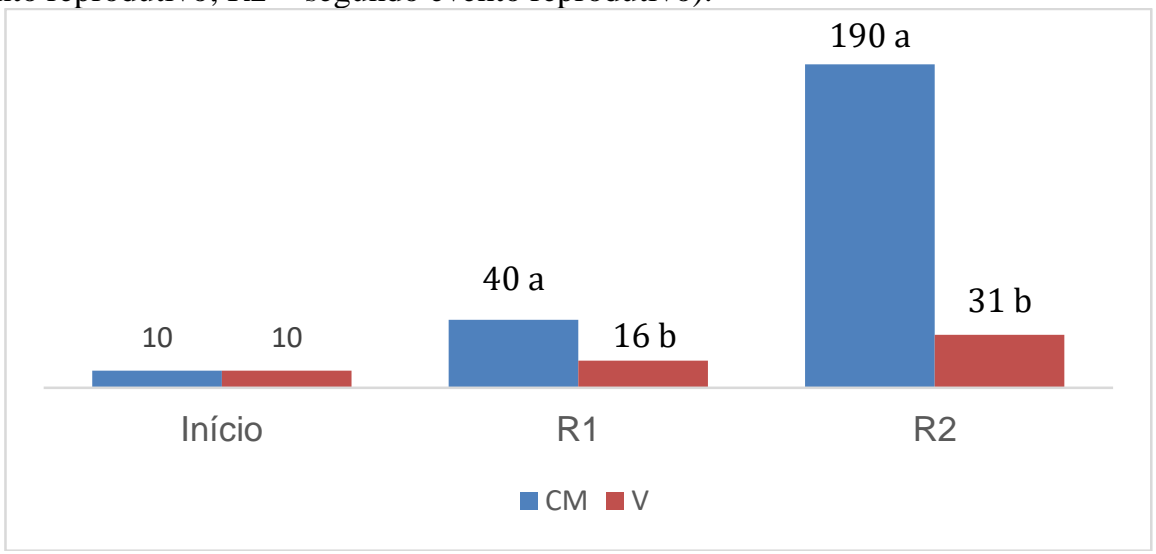

Fonte: Elaborado pelo autor.

Gráfico 2 - Crescimento populacional de Simocephalus iheringi alimentado com cultura mista (CM) e com Volvox sp. (V) $(\mathrm{R} 1=$ primeiro evento reprodutivo; $\mathrm{R} 2$ = segundo evento reprodutivo).

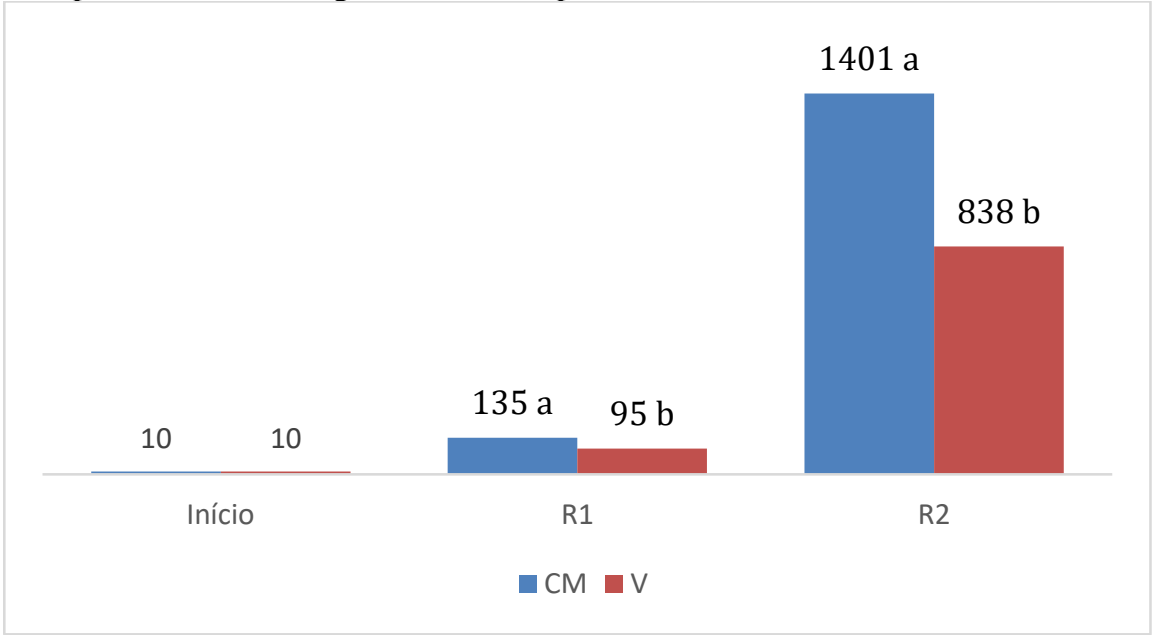

Fonte: Elaborado pelo autor.

A fecundidade também foi maior, com a dieta mista, para ambas as espécies, observando-se aumento da fecundidade da Ceriodaphnia dubia e diminuição da fecundidade do Simocephalus iheringi, no segundo evento reprodutivo (Tabela 1).

Tabela 1 - Número de ovos por fêmea em função da dieta e do tamanho da população (R1 = primeiro evento reprodutivo; $\mathrm{R} 2$ = segundo evento reprodutivo)

\begin{tabular}{ccccc} 
Dieta & \multicolumn{2}{c}{ Ceriodaphnia dubia } & \multicolumn{2}{c}{ Simocephalus iheringi } \\
& $\mathrm{R} 1$ & $\mathrm{R} 2$ & $\mathrm{R} 1$ & $\mathrm{R} 2$ \\
$\mathrm{CM}$ & 3,50 & 3,80 & 13,50 & 10,38 \\
$\mathrm{~V}$ & 1,58 & 1,92 & 9,50 & 8,82
\end{tabular}

Fonte: Elaborada pelo autor.

As características relacionadas ao ciclo de vida foram pouco afetadas pela dieta. Para Ceriodaphnia dubia, o tempo de desenvolvimento embrionário, o tempo de desenvolvimento pósembrionário, o tempo do ciclo reprodutivo e o número de eventos reprodutivos foram iguais, nos dois 
tratamentos, observando-se alteração apenas no tempo de vida, que foi menor com a dieta unialgal. Para Simocephalus iheringi, o tempo de desenvolvimento pós-embrionário, o tempo do ciclo reprodutivo e o tempo de vida foram maiores, com a dieta unialgal, em relação à dieta mista (Tabela 2).

Tabela 2 - Ciclo de vida de Ceriodaphnia dubia e Simocephalus iheringi, em função da dieta $(\mathrm{CM}=$ cultura mista; $\mathrm{V}=$ Volvox sp; $\mathrm{TDE}=$ tempo de desenvolvimento embrionário; TDPE = tempo de desenvolvimento pós-embrionário; TCR = tempo do ciclo reprodutivo; NER = número de eventos reprodutivos; TV = tempo de vida).

\begin{tabular}{|c|c|c|c|c|c|c|c|c|c|c|}
\hline \multirow{2}{*}{ Dieta } & \multicolumn{5}{|c|}{ Ceriodaphnia dubia } & \multicolumn{5}{|c|}{ Simocephalus iheringi } \\
\hline & $\begin{array}{c}\text { TDE } \\
\text { (dias) }\end{array}$ & $\begin{array}{l}\text { TDPE } \\
\text { (dias) }\end{array}$ & $\begin{array}{c}\text { TCR } \\
\text { (dias) }\end{array}$ & NER & $\begin{array}{c}\text { TV } \\
\text { (dias) }\end{array}$ & $\begin{array}{l}\text { TDE } \\
\text { (dias) }\end{array}$ & $\begin{array}{l}\text { TDPE } \\
\text { (dias) }\end{array}$ & $\begin{array}{c}\mathrm{TCR} \\
\text { (dias) }\end{array}$ & NER & $\begin{array}{c}\text { TV } \\
\text { (dias) }\end{array}$ \\
\hline $\mathrm{CM}$ & 2 & 5 & 6 & 2 & 16 & 3 & 6 & 7 & 3 & 21 \\
\hline $\mathrm{V}$ & 2 & 5 & 6 & 2 & 14 & 3 & 7 & 8 & 3 & 28 \\
\hline
\end{tabular}

Fonte: Elaborada pelo autor.

A taxa intrínseca de crescimento e o tempo de duplicação da população sofreram variação, entre os tratamentos e entre os eventos reprodutivos (Tabela 3), conforme esperado, visto que essas variáveis são dependentes da fecundidade e do tempo do ciclo reprodutivo.

Tabela 3 - Taxa intrínseca de crescimento $\left(r=\right.$ indivíduos dia $\left.{ }^{-1}\right)$ e tempo de duplicação da população (d), em função dos eventos reprodutivos.

\begin{tabular}{|c|c|c|c|c|c|c|c|c|}
\hline \multirow{3}{*}{ Dieta } & \multicolumn{4}{|c|}{ Ceriodaphnia dubia } & \multicolumn{4}{|c|}{ Simocephalus iheringi } \\
\hline & \multicolumn{2}{|c|}{$\mathrm{R} 1$} & \multicolumn{2}{|c|}{$\mathrm{R} 2$} & \multicolumn{2}{|c|}{ R1 } & \multicolumn{2}{|c|}{$\mathrm{R} 2$} \\
\hline & $\mathrm{r}$ & d & $\mathrm{r}$ & $\mathrm{d}$ & $\mathrm{r}$ & d & $\mathrm{r}$ & $\mathrm{d}$ \\
\hline $\mathrm{CM}$ & 0,23 & 3,01 & 0,26 & 2,67 & 0,37 & 1,87 & 0,51 & 1,36 \\
\hline $\mathrm{V}$ & 0,079 & 8,77 & 0,11 & 6,30 & 0,28 & 2,48 & 0,38 & 1,82 \\
\hline
\end{tabular}

Fonte: Elaborada pelo autor.

A temperatura e o suprimento alimentar influenciam a duração do desenvolvimento embrionário e pós-embrionário, bem como o esforço reprodutivo em laboratório, em condições controladas. De modo geral, a duração do tempo de desenvolvimento decresce com o aumento de temperatura. A fecundidade das fêmeas está diretamente relacionada à oferta e à qualidade do alimento, bem como ao tamanho dos animais, geralmente aumentando nas espécies maiores.

O teor nutricional da dieta fornecida esse sim é o principal componente de interferência no ciclo, e a cultura multialgal houve mudanças totais no número de ovos produzidos e até mesmo no número de partenogênese. 
Por consequência dos processos biológicos a sobre carga populacional aumentava e então ocorria mortalidade total da cultura. Nutrição e diversos micronutrientes disponíveis em diferentes espécies de fitoplâncton é o determinante na influência da sobrevivência dos indivíduos.

Ceriodaphinia tem menor tempo de vida (alguns indivíduos com expectativa de 16/18 dias de vida), seu tempo de reprodução é com 5 dias, os neonatos após 5 dias já estão aptos a reprodução (porem podem começar a se reproduzir no $8^{\circ}$ dia de vida). O numero de partenogêneses nas condições do cultivo foi associado ao tipo de dieta fornecida.

Simocephalus iheringi chegam a viver 28 dias. Os animais após a troca de carapaça ocorre crescimento de seu tamanho, (segunda partenogênese) a idade da primeira reprodução ocorre no $6^{\circ}$ dia.

Assim como Bachion (1996) relata a utilização de organismos autóctones em testes de toxidade, o gênero Simocephalus sp. por apresentar morfologia filtradora é afetado diretamente pelas condições do meio, podendo incrementar a qualidade destes teste, uma vez que as condições de cultivo bem como as condições de realização dos testes são próximas as características do ambiente.

Como Lazzaro (1987) relata o estudo realizado do potencial da espécie do cladócero Moina sp. para aquicultura. A espécie Simocephalus iheringi é caracterizada por ter média duração do desenvolvimento embrionário, rápido desenvolvimento pós-embrionário, com período extensivo de 28 dias no ciclo de vida, o que define uma boa produtividade, confirmando a boa prática para aquicultura, garantindo a disponibilidade do alimento natural.

Maier (1994) diz que espécies grandes e pequenas exibem diferentes tendências em sua bionomia: espécies grandes produzem ninhadas maiores, com ovos maiores e um maior dimorfismo sexual do que espécies menores, embora estas últimas apresentem um maior tamanho relativo dos ovos. Isto implica em um menor período de tempo desde a eclosão até a maturidade, o que lhes proporciona vantagens competitivas em relação às espécies maiores, quando existe uma limitação de alimento.

Melão (1997) relata o efeito da temperatura sobre o desenvolvimento embrionário e pósembrionário de sete espécies de cladóceros, observando que o tempo de desenvolvimento embrionário e pós-embrionário decresce, com um aumento da temperatura, para todas as espécies estudadas. Nesse trabalho, a temperatura foi controlada, de modo que o seu efeito sobre o desenvolvimento dos organismos não foi evidenciado.

Uma população de microcrustáceos só pode ter um crescimento bem-sucedido se houver alimento adequado e em quantidade suficiente. Mudanças no tamanho, qualidade, composição e concentração necessária de alimento favorecem determinadas espécies em detrimento de outras (LE CREN; LOWE-MCCONNELL, 1980). Esse trabalho comprova que mudanças na composição e na concentração do alimento influenciam diretamente o desenvolvimento embrionário e pós-embrionário. 
Vijverberg (1989) salienta a necessidade de se trabalhar com algas na fase de crescimento exponencial e baixas concentrações de alimento (concentrações de $10^{5}$ ou $10^{6}$ células $/ \mathrm{mL}$ são indicadas para herbívoros), para que o meio de cultura se aproxime das condições ambientais, onde o alimento normalmente é limitado.

Foto 2 - Espécimes com diferentes aspectos morfológicos (polimorfismo), encontrados nos frascos de cultivo (a: padrão morfológico normal; b, c: indivíduos modificados).

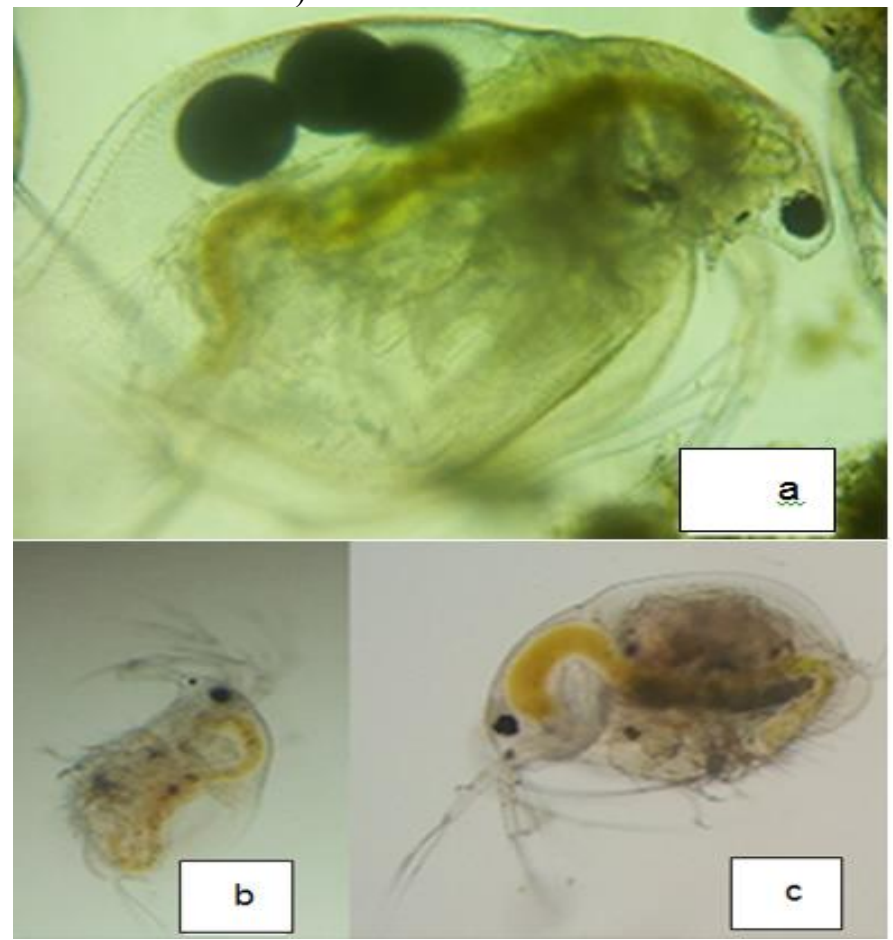

Fonte: O Autor.

Foto 3 - Fêmeas de Simocephalus iheringi com ovos e neonatos (a: fêmea com ovo de resistência; b: fêmea com ovos; c: fêmea com neonatos)

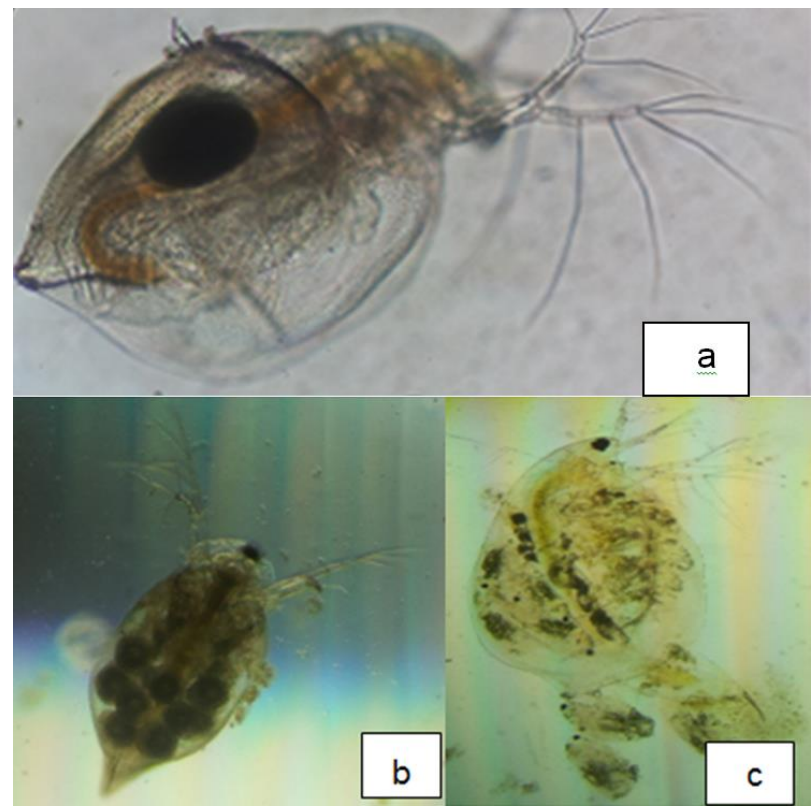

Fonte: O Autor. 


\section{CONCLUSÕES}

A fecundidade foi afetada pela dieta, tamanho das fêmeas (Ceridaphnia dubia) e pelo tamanho população (Simocephalus iheringi).

Os melhores resultados, para ambas as espécies, foram obtidos com a dieta constituída pela cultura mista. 


\section{REFERÊNCIAS}

BACHION, M.A. Estudo do crescimento e desenvolvimento populacional de três espécies zooplanctônicas submetidas a diferentes dietas alimentares. Jaboticabal: UNESP. 148p. (Dissertação) 1996.

ELMOOR-LOUREIRO, L.M.A. Manual de identificação de cladóceros límnicos do Brasil. Cladóceros límnicos - Brasil. I. Brasília. Universa - UCB, (1-156p), 1997.

LAZZARO, X. A review of planktivorous fishes: their evoluation, feeding behaviours, selectivities and impacts. Hydrobiologia, v. 146, p. 97-167, 1987.

LE CREN, E.D.; LOWE-MCCONNELL, R.H. The functioning of freshwater ecosystems. Cambridge: Cambridge University Press. 588 p. (IBP-Handbook, 22). 1980.

MAIER, G. Patterns of life history among cyclopoid copepods of central Europe. Freshwater Biology, v. 3, p. 77-86,1994.

MELÃO, M.G.G. A comunidade planctônica (fitoplâncton e zooplâncton) e produtividade secundária do zooplâncton de um reservatório oligotrófico. São Carlos: UFSCar, 152 p. (Tese).1997.

SANTEIRO, R. M.; PINTO-COELHO, R. M.; SIPAÚBA-TAVARES, L. H. Diurnal variation of zooplankton biochemical composition and biomass in plankton production on tanks. Acta Sci. Biol., v. 28, n. 2, p. 103-108, april/june, 2006.

SiPAÚBA-TAVAReS, L. H.; BACHION, M. A. Population growth and development of two species of Cladocera, Moina micrura and Diaphanosoma birgei, in laboratory. Braz. J. Biol., v. 62, n. 4A, p. 701-711, 2002.

VIJVERBERG, J. Culture techniques for studies on the growth, development and reproduction of copepods and cladocerans under laboratory and in situ conditions. Freshwater Biology, v. 21, p. 317-373, 1989.

ZANIBONI FILHO, E. Larvicultura de peixes de água doce. Belo Horizonte. Informe Agropecuário, v. 21, n. 203, p. 69-77, mar./abr., 2000. 